\title{
Diabetic Foot Ulcers in a Kenyan Referral and Teaching Hospital: Risk Factors, Patient Characteristics and Clinical Outcomes
}

\author{
Mutonga DM ${ }^{1^{*}}$, Mureithi MW ${ }^{2,3}$, Ngugi $\mathrm{NN}^{4}$ and Otieno $\mathrm{FC}^{5}$ \\ ${ }^{1}$ Institute of Tropical and Infectious Diseases, College of Health Sciences, University of Nairobi, Kenya \\ ${ }^{2}$ KAVI-Institute of Clinical Research, Kenya \\ ${ }^{3}$ Department of Medical Microbiology, College of Health Sciences, University of Nairobi, Kenya \\ ${ }^{4}$ Department of Medicine, Kenyatta National Hospital, Kenya \\ ${ }^{5}$ Department of Clinical Medicine and Therapeutics, College of Health Sciences, University of Nairobi, Kenya
}

"Correspondence: Daniel Munyambu Mutonga, Institute of Tropical and Infectious Diseases, College of Health Sciences, University of Nairobi, Kenya

Received on 18 July 2019; Accepted on 29 August 2019; Published on 03 September 2019

Copyright $\odot 2019$ Mutonga DM, et al. This is an open access article and is distributed under the Creative Commons Attribution License, which permits unrestricted use, distribution, and reproduction in any medium, provided the original work is properly cited.

\begin{abstract}
Introduction: The burden of diabetes mellitus (DM) is increasing in resource-poor settings leading to a rise in diabetic complications. Foot complications result in almost half of all hospital admissions among diabetic patients and may result in amputations or death.

Objective: To investigate the sociodemographic, clinic-laboratory characteristics and clinical outcomes of patients with diabetic foot ulcers (DFU) in a clinical setting.

Materials and Methods: A cross-sectional study of 84 adult consecutive inpatients and outpatients at Kenyatta National Hospital (KNH) with any type of DM and having active DFU was conducted over 12 months. History and physical examinations findings were recorded through a structured questionnaire. Relevant data on the most recent blood tests and clinical outcomes for patients with foot ulcers were retrieved from the patients' medical notes and analysed.

Results: Majority (68\%) were inpatients. The mean age was 60.30 years with $68 \%$ living in urban areas and $60 \%$ having minimal or no formal education. $8 \%$ were newly diagnosed with DM. The median duration of DM was 6.5 years. A majority (96\%) had type 2 diabetes mellitus (T2DM). $45 \%$ were on insulin only, $18 \%$ on oral drugs only and $32 \%$ on a combination of both. The median random blood sugar was $9.60 \mathrm{mmol} / \mathrm{L}$ and glycated haemoglobin was $8.80 \%$. Although $61 \%$ of patients had co-morbid hypertension, only about $40 \%$ had elevated systolic blood pressure (BP) while $23 \%$ had elevated diastolic pressures. A majority of the patients had good lipid profile, $85 \%$ with desirable total cholesterol and $70 \%$ having ideal low-density lipoproteins. The mortality rate among patients with DFU was $11 \%$.

Conclusion: There are poor outcomes for patients with DFU in this setting such as poor wound healing, high recurrence rates, increased amputations and mortality compared to previous studies. However, the prevalence of uncontrolled hypertension, dyslipidaemia and neuropathy was much lower than earlier local reports.
\end{abstract}


Keywords: diabetic foot ulcers, Kenya, risk factors, clinical outcomes

Abbreviations: BP: blood pressure; DFU: diabetic foot ulcers; DM: diabetes mellitus; IQR: interquartile range; KNH: Kenyatta National Hospital; T2DM: type 2 diabetes mellitus; UoN: University of Nairobi

\section{Introduction}

The global prevalence of DM is $8.8 \%$ [1]. By 2030, diabetes will have affected 188 million adults in their fourth and fifth decades and $80 \%$ of these patients will come from resource-poor countries [2]. Globally, foot complications result in $25-50 \%$ of all hospital admissions in DM patients [3].

In Africa, the prevalence of DFU ranges from 3.2-19.1\% [4-10]. A DFU is any foot with ulceration and linked to neuropathy and/or peripheral vascular disease in a patient with DM [11]. Previous reports reveal that the prevalence of diabetic polyneuropathy in Uganda, Kenya and Tanzania vary from 29.4-44\% [6, 7, 12, 13]. In addition, 40\% of diabetic patients in Nigeria present with the peripheral arterial disease [7]. The occurrence of diabetic amputations, a common complication of DM, varies in Sub-Saharan Africa but is generally thought to be low especially in the rural areas $[14,15]$. However, from recent accounts, the prevalence of lower limb amputations among DM patients ranges widely from $11.2-78 \%[4,5,16-19]$.

Global healthcare expenditure to treat and prevent DM was between USD 376 billion (2010) and 673 billion (2015) $[1,2]$. The estimated cost of treating a DFU in resource-rich countries is 6 to 10 times that in resource-poor countries revealing inequalities in access to health resources [20-22]. Moreover, DFU have mortality rates ranging from 2$14.3 \%$ in the African setting [4, 23-25).

There is limited data on DFU in Kenya. Previous local publications have looked at the prevalence, associated risk factors, types, and duration of DFU among the study participants [26-29]. There is no mention of outcomes on patients with DFU in the country. The present study aimed to investigate the sociodemographic, clinical characteristics and clinical outcomes of DFU among adult diabetic patients at $\mathrm{KNH}$.

\section{Materials and Methods}

These study methods were adapted from studies by Nyamu et al. and Chalya et al. [5, 27].

\section{Study design}

A hospital-based cross-sectional study was conducted over 12 months from September 2017 to August 2018 . Adult diabetic patients with active DFU were recruited by consecutive sampling from medical inpatient departments and the diabetes outpatient clinic at KNH, Nairobi, Kenya, this is the largest hospital in East and Central Africa.

\section{Data collection}

Basic patient and clinical characteristics were collected as part of the routine history taking and usual physical examinations. Patients' basic characteristics included age, sex, area of residence, and level of education. Clinical characteristics including presence of comorbidities, smoking history, alcohol use, duration of diabetes, type of diabetes, duration of DFU, current diabetes medications, previous history of healed foot ulcers, type of DFU, Wagner's classification, and location of the lesion were recorded for each patient. Peripheral neuropathy and peripheral vascular disease were also assessed. Height, weight and BP with the adult cuff standard technique were also measured.

The outcomes of the DFU were collected for patients at the end of the study by reviewing their medical records. Relevant information from the latest case notes, diabetic clinic or medical wards was extracted, classified [complete healing, non-healing, surgical intervention (minor or amputation) or mortality] and analysed. 


\section{Statistical analyses}

Microsoft $®$ Excel was used for data entry. Descriptive analyses was performed for all variables and data presented in charts and tables. Continuous variables such as age, time and, blood tests were converted into categorical variables using cut-off points. Independent two-tailed t-test was used to compare means, Chi-square test was used to verify the association of categorical variables and one-way ANOVA for comparison across multiple groups. Results were considered significant at $\mathrm{p}<0.05$. All analyses were performed using IBM® SPSS $®$ Statistical Package Version 23.0.

\section{Ethical considerations}

Approval was sought from the KNH-UoN Ethics and Research Committee (Approval Number P769/10/2016; website: http://erc.uonbi.ac.ke/) to conduct the study and patients enrolled only after informed consent was taken. Patient confidentiality and data security was guaranteed.

\section{Results}

Eighty-four patients were enrolled in the study (Figure 1). Most of the study patients were inpatients (68.4\%). The mean age was $60.30+12.88$ years. The youngest patient was 25 years while the oldest was 108 years, with most patients being in their fifth and sixth decades. The females were $54.1 \%$. Most of the patients $(68.3 \%)$ lived in urban areas while $31.7 \%$ lived in rural areas. A majority of $44.2 \%$ had primary education and $15.6 \%$ did not have any formal education. Males were more educated than females $(\mathrm{p}=0.001)($ Table 1$)$.

The median interquartile range (IQR) duration of DM was $6.5(1.25,12.5)$. A small proportion, $(8.5 \%)$ of patients were newly-diagnosed with diabetes, $17.3 \%$ had ever smoked cigarettes while $33.3 \%$ had ever taken alcohol. Males were more likely to be smokers $(\mathrm{p}=0.004)$. Fourteen patients $(16.5 \%)$ had no comorbidities, while the rest of the patients $(83.5 \%)$ had one or more comorbidities. The most common comorbidities were hypertension, kidney disease, heart disease and anemia. Most of the study patients had T2DM and were currently on medication (Table 2).

The mean BP was within normal ranges but 39.7\% had elevated systolic BP and 23.3\% had high diastolic BP. A large number of patients did not have recent laboratory results; $63.53 \%$ lacked HbA1c levels, $70 \%$ lacked lipid profile tests, 47\% lacked liver function tests and 30\% lacked kidney function tests. $39.3 \%$ of patients had high urea levels and $58.9 \%$ had elevated creatinine levels. Females had significantly better creatinine levels. A majority of the patients had desirable lipid profile tests (Table 3).

The median IQR duration of the DFU was $8(4,16)$ weeks. The longest duration of DFU was 312 weeks. Half of the patients had a previous history of DFU and a third had a prior history of amputation. At the time of the study, 77.6\% had recently used antibiotics to treat the ulcer. A majority (88.10\%) of the patients had ulcers on only one foot. Fortynine ulcers (53.26\%) were located on the right foot and 43 ulcers (46.74\%) on the left. Most ulcers were located on the forefoot and hindfoot. The DFU were mainly neuropathic or ischaemic and Wagner Stage 1 or 2. Of all the DFU, $54.3 \%$ had signs of diabetic neuropathy (Table 4).

The interval between the first and second interview was calculated and its median IQR was 98 (147) days. A majority of the patients were reviewed within 1-3 months after the first interview (28.9\%), 3-6 months (28.9\%) and 6-12 months $(21.1 \%)$. We reviewed 36 patients $(42.9 \%)$ while 48 patients $(57.1 \%)$ were lost to follow-up. A majority of the patients had good progress while $25 \%$ of the patients on follow-up had non-healing DFU. There was a mortality rate of $11.1 \%$ (Figure 2). 


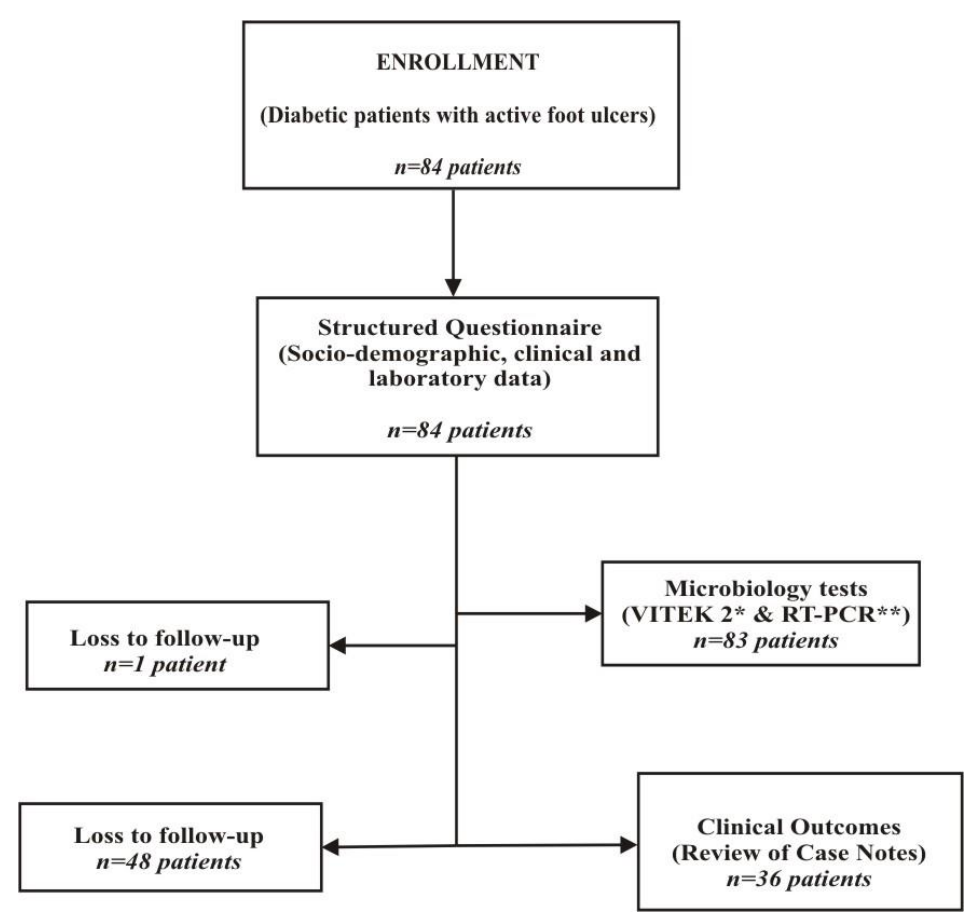

Figure 1: Flow chart of the study. The figure depicts the recruitment of patients into the study and study directed activities. Microbiological procedures and results are described in an earlier publication. "VITEK 2 machine was utilised for automated culture and sensitivity tests; ${ }^{* *}$ RT-PCR: real-time polymerase chain reaction.

\begin{tabular}{|c|c|c|c|c|c|}
\hline & & & & \multicolumn{2}{|l|}{ n (\%) } \\
\hline \multirow{2}{*}{\multicolumn{2}{|c|}{ Sex }} & \multicolumn{2}{|l|}{ Male } & \multicolumn{2}{|l|}{$39(45.9)$} \\
\hline & & \multicolumn{2}{|l|}{ Female } & \multicolumn{2}{|l|}{$46(54.1)$} \\
\hline \multirow{5}{*}{\multicolumn{2}{|c|}{ Age group }} & \multicolumn{2}{|l|}{$\leq 30$} & \multicolumn{2}{|l|}{$1(1.2)$} \\
\hline & & \multicolumn{2}{|l|}{$31-50$} & \multicolumn{2}{|l|}{$15(18.1)$} \\
\hline & & \multicolumn{2}{|l|}{$51-65$} & \multicolumn{2}{|l|}{$42(50.6)$} \\
\hline & & \multicolumn{2}{|l|}{$66-80$} & \multicolumn{2}{|l|}{$20(24.1)$} \\
\hline & & \multicolumn{2}{|l|}{$\geq 81$} & \multicolumn{2}{|l|}{$5(6.0)$} \\
\hline & & Total population & Male & Female & $p$ value \\
\hline Age (years) mean (SD) & & $60.30(12.88)$ & $58.62(11.38)$ & $61.65(13.95)$ & 0.29 \\
\hline \multirow{4}{*}{ Education level n (\%) } & None & $12(15.6)$ & $2(5.9)$ & $10(23.3)$ & \multirow{4}{*}{0.001} \\
\hline & Primary & $34(44.2)$ & $12(35.3)$ & $22(51.2)$ & \\
\hline & Secondary & $24(31.2)$ & $13(38.2)$ & $11(25.6)$ & \\
\hline & Tertiary & $7(9.1)$ & $7(20.6)$ & $0(0.0)$ & \\
\hline \multirow{2}{*}{ Residence n (\%) } & Urban & $56(68.3)$ & $29(78.4)$ & $27(60.0)$ & \multirow{2}{*}{0.097} \\
\hline & Rural & $26(31.7)$ & $8(21.6)$ & $18(40.0)$ & \\
\hline
\end{tabular}

Table 1: Patients' sociodemographic characteristics. This table shows the sociodemographic characteristics of the study patients. The lower part of the table shows differences in variables across gender and were considered statistically significant at $\mathrm{p}<0.05$. 


\begin{tabular}{|c|c|c|c|c|c|}
\hline & & & & \multicolumn{2}{|l|}{ n (\%) } \\
\hline \multirow{3}{*}{\multicolumn{2}{|c|}{ Type of diabetes mellitus $n(\%)$}} & \multicolumn{2}{|l|}{ Type $1 \mathrm{DM}$} & \multicolumn{2}{|l|}{$2(2.4)$} \\
\hline & & \multicolumn{2}{|l|}{ Type 2 DM } & \multicolumn{2}{|l|}{$81(96.4)$} \\
\hline & & \multicolumn{2}{|l|}{ Gestational DM } & \multicolumn{2}{|l|}{$1(1.2)$} \\
\hline \multirow{2}{*}{\multicolumn{2}{|c|}{ History of diabetes mellitus n (\%) }} & \multicolumn{2}{|c|}{ Yes (previously diagnosed) } & \multicolumn{2}{|l|}{$75(91.5)$} \\
\hline & & \multicolumn{2}{|c|}{ No (newly diagnosed) } & \multicolumn{2}{|l|}{$7(8.5)$} \\
\hline \multicolumn{2}{|c|}{ Duration of DM (years) median (IQR) } & \multicolumn{4}{|c|}{$6.5\left(11.25^{*}\right)$} \\
\hline \multirow{4}{*}{\multicolumn{2}{|c|}{ Duration of DM (years) n (\%) }} & \multicolumn{2}{|l|}{$<5$} & \multicolumn{2}{|l|}{$36(46.2)$} \\
\hline & & \multicolumn{2}{|l|}{$6-10$} & \multicolumn{2}{|l|}{$18(23.1)$} \\
\hline & & \multicolumn{2}{|l|}{$10-20$} & \multicolumn{2}{|l|}{$19(24.4)$} \\
\hline & & & \multicolumn{2}{|l|}{$5(6.4)$} \\
\hline \multirow{6}{*}{\multicolumn{2}{|c|}{ Type of treatment $\mathrm{n}(\%)$}} & \multicolumn{2}{|l|}{$\begin{array}{l}>21 \\
\text { None }\end{array}$} & \multicolumn{2}{|l|}{$2(2.4)$} \\
\hline & & \multicolumn{2}{|l|}{ Diet } & \multicolumn{2}{|l|}{$1(1.2)$} \\
\hline & & \multicolumn{2}{|l|}{$\mathrm{OHA}$} & \multicolumn{2}{|l|}{$15(17.6)$} \\
\hline & & \multicolumn{2}{|l|}{ Insulin } & $38(44.7)$ & \\
\hline & & Both diet and $\mathrm{OH}$ & & $2(2.4)$ & \\
\hline & & Both OHA and ins & & $27(31.8)$ & \\
\hline Risk factors & & Total population & Male & Female & p value \\
\hline Smokino habits $\mathrm{n}(\%)$ & Yes & $13(17.3)$ & $11(31.4)$ & $2(5.0)$ & 0.004 \\
\hline & No & $62(82.7)$ & $24(68.6)$ & $38(95.0)$ & \\
\hline Alcobol intake n $(O)$ & Yes & $25(33.3)$ & $18(51.4)$ & $7(17.5)$ & 0003 \\
\hline Aiconor milake in (\%) & No & $50(66.7)$ & $17(48.6)$ & $33(82.5)$ & 0.000 \\
\hline & None & $14(16.5)$ & $7(17.9)$ & $7(15.2)$ & \\
\hline & 1 & $33(38.8)$ & $15(38.5)$ & $18(39.1)$ & \\
\hline & 2 & $19(22.4)$ & $7(17.9)$ & $12(26.1)$ & \\
\hline Number of comorbidities n (\%) & 3 & $13(15.3)$ & $5(12.8)$ & $8(17.4)$ & 0.509 \\
\hline & 4 & $5(5.9)$ & $4(10.3)$ & $1(2.2)$ & \\
\hline & 5 & $1(1.2)$ & $1(2.6)$ & $0(0.0)$ & \\
\hline
\end{tabular}

Table 2: Clinical characteristics from patients' history. This table represents descriptive statistics of the clinical findings from patients' medical history, where both mean and median were reported, the data was not normally distributed. Differences across gender were statistically significant when $\mathrm{p}<0.05$.

\begin{tabular}{|l|l|l|l|l|l|}
\hline Parameter & Total mean (SD) & Total median (IQR) & Male & Female & $\mathrm{p}$ value \\
\hline $\begin{array}{l}\text { Systolic blood pressure } \\
(\mathrm{mmHg})\end{array}$ & $136.05(34.51)$ & & $138.25(34.72)$ & $138.39(24.77)$ & 0.985 \\
\cline { 1 - 3 } $\begin{array}{l}\text { Diastolic blood pressure } \\
(\mathrm{mmHg})\end{array}$ & $80.08(18.74)$ & & $80.56(19.47)$ & $80.61(12.41)$ & 0.991 \\
\hline RBS $^{1}(\mathrm{mmol} / \mathrm{L})$ & $14.12(11.03)$ & $9.60(8.95)$ & $10.08(4.96)$ & $11.57(7.28)$ & 0.373 \\
\hline $\mathrm{HbA1c}^{2}(\%)$ & $8.40(2.29)$ & $8.80(2.80)$ & $9.59(3.75)$ & $8.51(1.74)$ & 0.358 \\
\hline Urea $(\mathrm{mmol} / \mathrm{L})^{\text {Creat }^{3}(\mathrm{mmol} / \mathrm{L})}$ & $12.05(10.58)$ & $7.1(11.85)$ & $19.36(20.90)$ & $9.67(16.68)$ & $\mathbf{0 . 0 4 9}$ \\
\hline LDL-C $^{4}(\mathrm{mmol} / \mathrm{L})$ & $147.53(74.06)$ & $114.55(111.2)$ & $228.19(156.38)$ & $\begin{array}{l}133.12 \\
(100.72)\end{array}$ & $\mathbf{0 . 0 1 5}$ \\
\hline Albumin $(\mathrm{g} / \mathrm{L})$ & $1.40(0.34)$ & $1.51(0.81)$ & $1.65(0.90)$ & $1.82(0.79)$ & 0.627 \\
\hline
\end{tabular}

Table 3: Clinical and laboratory parameters. This table represents descriptive statistics of the clinical and laboratory parameters from physical examination and reviewing of patients' clinical records, where both mean and median were reported, the data was not normally distributed. Statistically significant $p<0.05$. ${ }^{1}$ Random blood sugar ${ }^{2}$ Glycated hemoglobin ${ }^{3}$ Creatinine ${ }^{4}$ Low-density lipoprotein-cholesterol. 


\begin{tabular}{|c|c|c|c|c|}
\hline & & & \multicolumn{2}{|l|}{$\mathrm{n}(\%)$} \\
\hline \multicolumn{5}{|c|}{ Duration of DFU (weeks) $8\left(12^{*}\right)$} \\
\hline \multirow{4}{*}{\multicolumn{2}{|c|}{ Duration of DFU (weeks) n (\%) }} & $>6$ & \multicolumn{2}{|l|}{$27(37.5)$} \\
\hline & & $7-26$ & \multicolumn{2}{|l|}{$38(52.8)$} \\
\hline & & $27-52$ & \multicolumn{2}{|l|}{$7(9.7)$} \\
\hline & & $>52$ & \multicolumn{2}{|l|}{$0(0.0)$} \\
\hline \multirow{2}{*}{\multicolumn{2}{|c|}{ History of previous DFU n (\%) }} & Yes & \multicolumn{2}{|l|}{$40(50)$} \\
\hline & & No & \multicolumn{2}{|l|}{$40(50)$} \\
\hline \multirow{2}{*}{\multicolumn{2}{|c|}{ History of previous amputation $\mathrm{n}(\%)$}} & Yes & \multicolumn{2}{|l|}{$26(33.3)$} \\
\hline & & No & \multicolumn{2}{|l|}{$52(66.7)$} \\
\hline \multirow{2}{*}{\multicolumn{2}{|c|}{ History of any recent antibiotic use $\mathrm{n}(\%)$}} & Yes & \multicolumn{2}{|l|}{$45(77.6)$} \\
\hline & & No & \multicolumn{2}{|l|}{$13(22.4)$} \\
\hline & & Right & Left & Total \\
\hline \multirow{6}{*}{$\begin{array}{l}\text { Anatomic site of foot ulcer } \\
\mathrm{n}(\%)\end{array}$} & Forefoot & $25(51.0)$ & $24(55.8)$ & $49(53.3)$ \\
\hline & Midfoot & $7(14.3)$ & $2(4.7)$ & $9(9.8)$ \\
\hline & Hindfoot & $11(22.4)$ & $16(37.2)$ & $27(29.4)$ \\
\hline & Forefoot \& midfoot & $4(8.2)$ & $0(0.0)$ & $4(4.4)$ \\
\hline & Midfoot \& hindfoot & $1(2.0)$ & $0(0.0)$ & $1(1.1)$ \\
\hline & Forefoot, midfoot \& hindfoot & $1(2.0)$ & $1(2.3)$ & $2(2.2)$ \\
\hline \multirow[t]{3}{*}{ Type of ulcer n (\%) } & Ischaemic & $20(40.8)$ & $22(51.2)$ & $42(45.6)$ \\
\hline & Neuropathic & $25(51.0)$ & $18(41.9)$ & $43(46.7)$ \\
\hline & Neuroischaemic & $4(8.2)$ & $3(7.0)$ & $7(7.6)$ \\
\hline \multirow{6}{*}{ Wagner stage n $(\%)$} & 0 & $1(2.0)$ & $0(0.0)$ & $1(1.1)$ \\
\hline & 1 & $11(22.4)$ & $16(37.2)$ & $27(29.4)$ \\
\hline & 2 & $16(32.7)$ & $12(27.9)$ & $28(30.4)$ \\
\hline & 3 & $10(20.4)$ & $9(20.9)$ & $19(20.7)$ \\
\hline & 4 & $10(20.4)$ & $4(9.3)$ & $14(15.2)$ \\
\hline & 5 & $1(2.0)$ & $2(4.7)$ & $3(3.3)$ \\
\hline
\end{tabular}

Table 4: Clinical characteristics of the DFU. This table represents duration, types, location and other clinical characteristics of DFU among the study patients. "The interquartile range of duration of DFU was from 4 to 16. 


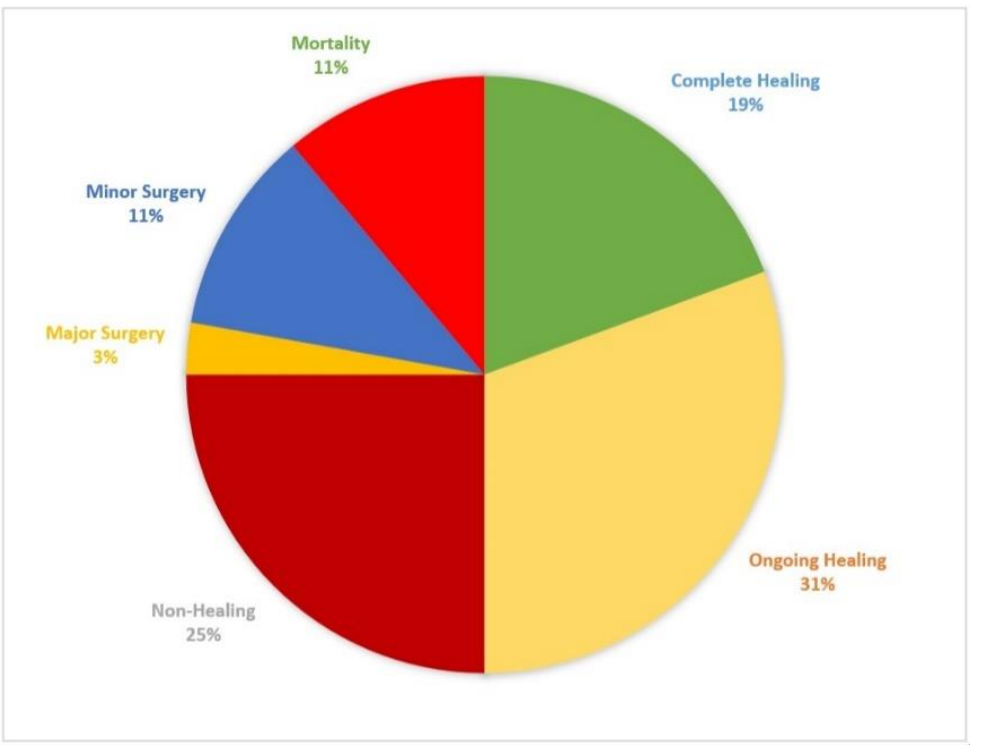

Figure 2: Distribution of clinical outcomes of DFU for the study patients. The chart depicts the proportion of study patients reviewed with a particular outcome of interest. Complete and ongoing healing were considered to be good outcomes while non-healing wounds and mortality as poor outcomes.

\section{Discussion}

Africa is the second most common region to be affected by DFU according to a recent global meta-analysis [30]. In the present study, there was a high recurrence rate of DFU (50\%), comparable to facilities that follow best practices (70\%) [31]. This warrants further investigation to see if patients who have had prior DFU adhere to proper foot care.

The mean age of patients in this study was slightly higher than in earlier studies [5, 27, 29]. Most of the study patients were however in their fifth and sixth decades just like in other studies in East Africa [5, 8, 9, 28,29]. There were more females than males enrolled in this present study similar to an earlier study in Kenya but in contrast to other studies in Sudan, Tanzania, and Ethiopia where males were more [5, 8, 27, 28, 32].

In the present study, about $60 \%$ had minimal or no formal education similar to studies in Sudan and Tanzania [5, 32]. Moreover, a majority of patients in this study were from urban areas similar to publications from Sudan and Ethiopia [9, 10,32]. A study in Tanzania also enrolled patients mainly from rural areas [5]. In this current study, more patients were from urban areas mainly because of KNH's location in Nairobi.

Mariam et al. reported T2DM to be a significant risk factor for developing a DFU [9]. In this present study, almost all of the patients had T2DM. A small proportion of the study patients were newly-diagnosed with DM comparable to an earlier study [27]. The median duration of DM was 6.5 years, revealing that DFU are developing much earlier than before. This could be an indicator of poor foot care or inadequate foot care.

In the present study, only a small proportion of patients were not on medication. A majority were on insulin therapy, whether alone or in combination with oral drugs. The high number of patients on insulin could result from the fact that inpatients often have deranged glucose levels and require insulin for strict glucose control. However, less than half of the current study patients were on insulin therapy only which is comparable to earlier studies in Kenya and Ethiopia [9, 27]. Although poor glycaemic control often leads to DFU, insulin treatment is in itself a risk factor for developing DFU in Tanzania [6, 27, 29]. The glycemic control based on HbAlc level for patients in this present study was similar to an earlier study in KNH but much worse than in a recent study in rural Kenya [27, 29].

The prevalence of uncontrolled hypertension in this study was much lower than an earlier study in Kenya [29]. In the current study, the rates of kidney disease were similar to findings in Ethiopia, while heart disease was higher than in 
Sudan $[8,32]$. In this present study, the lipid profile was much better than that reported by Nyamu et al. and could be a result of improved patient awareness or better management of dyslipidaemia by the clinicians [27].

The median duration of DFU was 8 weeks in this current study. This was shorter than in earlier studies in Kenya and Tanzania and could perhaps indicate increased patient awareness, increased foot care, and increased foot care assessment by the clinicians [5, 27]. DFU is often undiagnosed because of both patient or clinician related factors [33]. It is important that patients examine their feet regularly and clinicians not to omit to ask or examine for the diabetic foot.

Neuropathy is a significant risk factor for developing DFU [6, 9]. Peripheral neuropathy usually begins with autonomic dysfunction, then sensory nerves are affected and finally motor nerves deteriorate [31]. Although the prevalence of neuropathy in this present study is much lower than in earlier Kenyan studies [27, 28], diabetic polyneuropathy among the study patients was much higher than in Uganda and Nigeria [7, 12]. This confirms a higher rate of neuropathy among Kenyan patients. A majority of the DFU in this present study was in Wagner Stage 1 and 2 in contrast to studies in Tanzania and Libya [5, 24]. This could also indicate an early presentation of the patients to this hospital.

In the present study, a third of the study patients had a prior amputation, which was thrice the rate of previous amputation in Tanzania [5]. However, after follow-up, only 14\% of patients had a surgical procedure compared to 90\% in Tanzania, mainly because the current study was conducted in medical and not surgical departments [5]. Half of the patients followed-up in this present study had good clinical outcomes. The mortality rate in this present study was high and similar to the study in Tanzania and a literature review from 19 African countries $[4,5]$. Mortality in Tanzania was associated with diabetes complications and advanced DFU [5]. In this present study, there was a nonhealing rate of $25 \%$ among the study patients who were followed-up. Healing of wounds is a complex immunological response to injury $[20,31,33]$.

\section{Limitations}

One of the limitations in this study is that funding for this study was allocated towards microbiological tests to isolate the bacteria from DFU as presented in an earlier publication [34]. As such, we relied on extraction of clinical data from patients medical records for retrieval of laboratory tests. Moreover, outcome of DFU in some instances depended on the review of medical notes and not repeat physical examinations.

\section{Conclusion}

There are poor outcomes for patients with DFU in this setting such as poor wound healing, high recurrence rates, increased lower-limb amputations and mortality compared to previous studies. The study patients were slightly older, female less educated but urbanised. The study patients had better control of hypertension and dyslipidaemia, lower levels of neuropathy and earlier presentation of DFU reflecting increased patient awareness and better management by clinicians. Majority of the patients in this study were on insulin and antibiotics.

\section{Recommendations}

We recommend the following:

- The high prevalence of poor outcomes for patients with DFU warrants the need to investigate biopsychosocial risk factors.

- Screening and foot assessment should be encouraged during each clinical visit. Examination focuses on peripheral neuropathy, peripheral arterial disease and plantar pressure [31]. Certain professional organisations recommend different prevention measures based on the patients' risk levels [20]. Patients with DM should be educated on smoking cessation and foot-care.

- Patients with DM should have laboratory tests such as UECs, LFTs, Lipid profile, and HbA1c every 6 months to rule out and properly manage diabetic complications.

\section{Conflicts of Interest}

The authors declare that they have no competing interests. 


\section{Funding Statement}

DMM received partial funding from KNH Research and Programs Department (KNH Study Registration Number: Med/101/2017) to conduct this study (website: http://knh.or.ke/). Most of the funding sought was for microbiology tests and PCR tests presented in an earlier publication. The funders had no role in study design, data collection and analysis, decision to publish or preparation of the manuscript.

\section{Acknowledgements}

We are grateful to all patients who participated in this study. Our sincere thanks goes towards the nursing staff in the Medicine Department and the Diabetes and Endocrinology Outpatient Clinic at Kenyatta National Hospital for their support. We also acknowledge Mr. Wycliffe Ayieko for his assistance in data analysis and Dr. Jackline Ngigi for her assistance in data collection.

\section{References}

1. IDF Diabetes Atlas 7th Edition (2015).

2. IDF Diabetes Atlas 4th Edition (2009).

3. Ezera Agwu, Ephraim O. Dafiewhare and Peter E. Ekanem (December 9th 2011). Possible Diabetic-Foot Complications in Sub-Saharan Africa, Global Perspective on Diabetic Foot Ulcerations, Thanh Dinh, IntechOpen.

4. Rigato M, Pizzol D, Tiago A, et al. Characteristics, prevalence, and outcomes of diabetic foot ulcers in Africa. A systemic review and meta-analysis. Diabetes Res Clin Pract. 2018;142(1):63-73.

5. Chalya PL, Mabula JB, Dass RM, et al. Surgical management of diabetic foot ulcers: A Tanzanian university teaching hospital experience. BMC Res Notes. 2011;4(365):1-7.

6. Chiwanga FS, Njelekela MA. Diabetic foot: prevalence, knowledge, and foot self-care practices among diabetic patients in Dar es Salaam, Tanzania - a cross-sectional study. J Foot Ankle Res. 2015;8(20):1-7.

7. Ogbera AO, Adeleye O, Solagberu B, et al. Screening for peripheral neuropathy and peripheral arterial disease in persons with diabetes mellitus in a Nigerian University Teaching Hospital. BMC Res Notes. 2015;8(533):1-6.

8. Deribe B, Woldemichael K, Nemera G. Prevalence and factors Influencing diabetic foot ulcer among diabetic patients attending Arbaminch Hospital, South Ethiopia. J Diabetes Metab. 2014;5(1):1-7.

9. Mariam TG, Alemayehu A, Tesfaye E, et al. Prevalence of diabetic foot ulcer and associated factors among adult diabetic patients who attend the diabetic follow-up clinic at the University of Gondar Referral Hospital, North West Ethiopia, 2016: Institutional-Based Cross-Sectional study. J Diabetes Res. 2017;2017(2879249):1-8.

10. Awadalla H, Noor SK, Elmadhoun WM, et al. Diabetes complications in Sudanese individuals with type 2 diabetes: Overlooked problems in sub-Saharan Africa? Diabetes Metab Syndr. 2017;11(S2):S1047-S51.

11. Alexiadou K, Doupis J. Management of diabetic foot ulcers. Diabetes Ther. 2012;3(1):1-15.

12. Kisozi T, Mutebi E, Kisekka M, et al. Prevalence, severity and factors associated with peripheral neuropathy among newly diagnosed diabetic patients attending Mulago hospital: a cross-sectional study. Afr Health Sci. 2017;17(2):463-73.

13. Mugambi-Nturibi E, Otieno CF, Kwasa T, et al. Stratification of persons with diabetes into risk categories for foot ulceration. East Afr Med J. 2009;86(5):233-39. 
14. Abbas ZG, Archibald LK. Epidemiology of the diabetic foot in Africa. Med Sci Monit. 2005;11(8):262-70.

15. Kengne AP, Choukem SP, Dehayem YM, et al. Diabetic foot ulcers in Cameroon: can microflora prevalence inform probabilistic antibiotic treatment? J Wound Car. 2006;15(8):363-66.

16. Verla TG. Terence. Diabetes related amputations in the north-west region of Cameroon. 2011.

17. Essoh JBS, Kodo M, Djè VDB, et al. Limb amputations in adults in an Ivorian Teaching Hospital. Niger J Clin Pract. 2009;12(3):245-47.

18. Nwosu C, Babalola MO, Ibrahim MH, et al. Major limb amputations in a tertiary hospital in North Western Nigeria. Afr Health Sci. 2017;17(2):508-12.

19. Udeabor SE, Adisa AO, Orlowska A, et al. Tumor-associated macrophages, angiogenesis, and tumor cell migration in oral squamous cell carcinoma. Ann Afr Med. 2017;16(4):181-85.

20. Singh N, Armstrong DG, Lipsky BA. Preventing foot ulcers in patients with diabetes. JAMA. $2005 ; 293(2): 217-28$.

21. Kasiya MM, Mang'anda GD, Heyes S, et al. The challenge of diabetic foot care: Review of the literature and experience at Queen Elizabeth Central Hospital in Blantyre, Malawi. Malawi Med J. 2017;29(2):218-23.

22. Danmusa UM, Terhile I, Nasir IA, et al. Prevalence and healthcare costs associated with the management of diabetic foot ulcer in patients attending Ahmadu Bello University Teaching Hospital, Nigeria. Int J Health Sci (Qassim). 2016;10(2)219-28.

23. Edo AE, Edo GO, Ezeani IU. Risk factors, ulcer grade and management outcome of diabetic foot ulcers in a Tropical Tertiary Care Hospital. Niger Med J. 2013;54(1):59-63.

24. Benkhadoura M, Alswehly M, Elbarsha A. Clinical profile and surgical management of diabetic foot in Benghazi, Libya. Foot Ankle Surg. 2016;22(1):55-58.

25. Foryoung JB, Ditah C, Fon PN, et al. Long-term mortality in outpatients with type 2 diabetes in a reference hospital in Cameroon: A retrospective cohort study. BMJ Open. 2018;8(2):1-7.

26. McLigeyo, Otieno LS. Diabetic ulcers--a clinical and bacteriological study. East Afr Med J. 1991;68(3):20410 .

27. Nyamu PN, Otieno CF, Amayo EO, et al. Risk factors and prevalence of diabetic foot ulcers at Kenyatta National Hospital , Nairobi. East Afr Med J. 2003;80(1):36-43.

28. Obimbo MM, Bundi PK, Collis F, et aL. Foot complications among diabetics attending a district hospital in Kenya : Predisposing factors and possible intervention. Ann Afr Surg. 2008;2(6):3-8.

29. Kibachio J, Omolo J, Muriuki Z, et al. Risk factors for diabetic foot ulcers in type 2 diabetes: a case control study, Nyeri, Kenya. Afr J Diab Med. 2013;21(1):1-4.

30. Zhang P, Lu J, Jing Y, et al. Global epidemiology of diabetic foot ulceration: a systematic review and metaanalysis. Ann Med. 2017;49(2):106-16.

31. Mendes JJ, Neves J. Diabetic foot infections: current diagnosis and treatment. J Diab Foot Complicat. 2012;4(2):26-45.

32. Shigidi M, Abdelgafar H, Taha ES. Awareness regarding diabetes control and diabetic nephropathy among Sudanese adults admitted with diabetic foot: a cross-sectional study. Pan Afr Med J. 2013;16(157):1-6. 
33. Bruhn-Olszewska B, Korzon-Burakowska A, Gabig-Cimińska M, et al. Molecular factors involved in the development of diabetic foot syndrome. Acta Biochim Pol. 2012;59(4):507-13.

34. Mutonga DM, Mureithi MW, Ngugi NN, et al. Bacterial isolation and antibiotic susceptibility from diabetic foot ulcers in Kenya using microbiological tests and comparison with RT- PCR in detection of S. aureus and MRSA. BMC Res Notes. 2019;12(1)1-6. 